\title{
Randomized algorithm of invariant zeros evaluation of electrical power system, defined in descriptor form
}

\author{
Magomed Gadzhiev ${ }^{1}$, Misrikhan Misrikhanov ${ }^{1}$, Vladimir Ryabchenko ${ }^{1}$, and Nikita Vasilenko ${ }^{1}$ \\ ${ }^{1}$ National research university "MPEI", Institute of Electrical Power Engineering, 14 Krasnokazarmennaya St, Moscow, Russia
}

\begin{abstract}
A randomized algorithm for computing the invariant zeros of the electric energy system as a dynamical system with many inputs and many outputs (MIMO system), specified in the descriptor form, is proposed. Definitions of invariant zeros are carried out by randomizing the original MIMO system and it reduces to a generalized eigenvalue problem for a numerical matrix. The application of the algorithm is illustrated by the example of calculating the invariant zeros of the linear model of the United Power System.
\end{abstract}

\section{Introduction}

The main object of practical calculations is the linearization of nonlinear descriptor mathematical model. Thus, equations [8] are considered for nonlinear dynamic system.

$$
\begin{aligned}
\frac{d \mathbf{x}}{d t} & =\mathbf{f}(\mathbf{x}, \mathbf{z}, \mathbf{u}), \\
\mathbf{0} & =\mathbf{g}(\mathbf{x}, \mathbf{z}, \mathbf{u}), \\
\mathbf{y} & =\mathbf{h}(\mathbf{x}, \mathbf{z}),
\end{aligned}
$$

where $\mathbf{x} \in \mathbf{R}^{n_{x}}$ - state variables vector; $\quad \mathbf{z} \in \mathbf{R}^{n_{z}}$ parameter vector; $\mathbf{u} \mathrm{O} \mathbf{R}^{r}$ - input stimulus vector; $\mathbf{u} \in \mathbf{R}^{r}$ - output quantity vector. Formula for vector functions $\mathbf{f}, \mathbf{g}$ and $\mathbf{h}$ is defined by system element mathematical models, measurement and correcting units.

Standard approach to llinearization (1), (2) connects with the procedure of Taylor expansion of vector functions $\mathbf{f}$ and $\mathbf{g}$ in small neighbourhood of steadystate process with $\mathbf{x}_{0}, \mathbf{z}_{0}, \mathbf{u}_{0}$ coordinates.

$$
\begin{gathered}
\mathbf{f}(\mathbf{x}, \mathbf{z}, \mathbf{u}) \approx \mathbf{f}\left(\mathbf{x}_{0}, \mathbf{z}_{0}, \mathbf{u}_{0}\right)+\frac{\partial \mathbf{f}}{\partial \mathbf{x}}{ }_{0} \Delta \mathbf{x}+\frac{\partial \mathbf{f}}{\partial \mathbf{z}} 0 \mathbf{z}_{0}+\frac{\partial \mathbf{f}}{\partial \mathbf{u}}{ }_{0} \Delta \mathbf{u}, \\
\mathbf{g}(\mathbf{x}, \mathbf{z}, \mathbf{u}) \approx \mathbf{g}\left(\mathbf{x}_{0}, \mathbf{z}_{0}, \mathbf{u}_{0}\right)+\frac{\partial \mathbf{g}}{\partial \mathbf{x}}{ }_{0} \Delta \mathbf{x}+\frac{\partial \mathrm{g}}{\partial \mathbf{z}}{ }_{0} \Delta \mathbf{z}+\frac{\partial \mathbf{g}}{\partial \mathbf{u}}{ }_{0} \Delta \mathbf{u}, \\
\mathbf{h}(\mathbf{x}, \mathbf{z}) \approx \mathbf{h}\left(\mathbf{x}_{0}, \mathbf{z}_{0}\right)+\frac{\partial \mathbf{h}}{\partial \mathbf{x}_{0}} \Delta \mathbf{x}+\frac{\partial \mathbf{h}}{\partial \mathbf{z}}{ }_{0} \Delta \mathbf{z}
\end{gathered}
$$

where $\Delta \mathbf{x}=\mathbf{x}-\mathbf{x}_{0} \sqcap x^{\prime} \in \mathbf{R}^{n_{x}}, \Delta \mathbf{z}=\mathbf{z}-\mathbf{z}_{0} \sqcap x^{\prime \prime} \in \mathbf{R}^{n_{z}}$, $\Delta \mathbf{u}=\mathbf{u}-\mathbf{u}_{0}\left\ulcorner u \in \mathbf{R}^{r}, \Delta \mathbf{y}=\mathbf{y}-\mathbf{y}_{0} \sqcap y \in \mathbf{R}^{m}-\right.$ deviation of corresponded vectors; $(*)_{0}-$ corresponded partial derivative, estimated in coordinates chosen.

Equation linearization (1) - (3) at a point of steady state motion gives linear descriptor (algebra-differential) equations

$$
\frac{d x^{\prime}}{d t}=\frac{\partial \mathbf{f}}{\partial \mathbf{x}} x_{0}^{\prime}+\frac{\partial \mathbf{f}}{\partial \mathbf{z}} x^{\prime \prime}+\frac{\partial \mathbf{f}}{\partial \mathbf{u}_{0}} u,
$$

$$
\begin{gathered}
0=\frac{\partial \mathbf{g}}{\partial \mathbf{x}}_{0} x^{\prime}+\frac{\partial \mathbf{g}}{\partial \mathbf{z}} x_{0}^{\prime \prime}+\frac{\partial \mathbf{g}}{\partial \mathbf{u}}{ }_{0} u, \\
y=\frac{\partial \mathbf{h}}{\partial \mathbf{x}} x_{0} x^{\prime}+\frac{\partial \mathbf{h}}{\partial \mathbf{z}}{ }_{0} x^{\prime \prime} .
\end{gathered}
$$

Hereafter for the purpose simplification of equations writing, index «0» of partial derivative is omitted.

When Jacobi matrix is invertible, equations (7), (8) can be reorganized into state space form

$$
\begin{aligned}
\frac{d x^{\prime}}{d t} & =\left(\frac{\partial \mathbf{f}}{\partial \mathbf{x}}-\frac{\partial \mathbf{f}}{\partial \mathbf{z}} \frac{\partial \mathbf{g}}{\partial \mathbf{z}}^{-1} \frac{\partial \mathbf{g}}{\partial \mathbf{x}}\right) x^{\prime}+\left(\frac{\partial \mathbf{f}}{\partial \mathbf{u}}-\frac{\partial \mathbf{f}}{\partial \mathbf{z}} \frac{\partial \mathbf{g}}{\partial \mathbf{z}}{ }^{-1} \frac{\partial \mathbf{g}}{\partial \mathbf{u}}\right) u \\
y & =\left(\frac{\partial \mathbf{h}}{\partial \mathbf{x}}-\frac{\partial \mathbf{h}}{\partial \mathbf{z}} \frac{\partial \mathbf{g}}{\partial \mathbf{z}}^{-1} \frac{\partial \mathbf{g}}{\partial \mathbf{x}}\right) x^{\prime}-\frac{\partial \mathbf{h}}{\partial \mathbf{z}} \frac{\partial \mathbf{g}}{\partial \mathbf{z}}^{-1} \frac{\partial \mathbf{g}}{\partial \mathbf{u}} u .
\end{aligned}
$$

If matrix $\frac{\partial \mathrm{g}}{\partial \mathbf{z}}$ is singular, then conversion to equations (10) will not exist. However, even in case of invertibility of $\frac{\partial \mathrm{g}}{\partial \mathbf{z}}$, the problem of conditionality of the matrix happens in practical calculations. For condition number

$$
\text { cond } \frac{\partial \mathbf{g}}{\partial \mathbf{z}} \gg 1
$$

of matrix $\frac{\partial \mathbf{g}}{\partial \mathbf{z}}$ is close to singular matrix and mathematical model (10) becomes «stiff» in relation to minute calculation errors and variation of parameters. To put it differently, the mathematical model (10) in case of correctness of inequation (11) is ill-defined from A.N. Tihonov [1,8].

If additional measures aren't taken, all calculations using the model (10), including controllability and observability analysis, will lead to serious errors. However, generally the method given comes across number of significant difficulties. Nonstrictly speaking, improvement of condition of matrix $\frac{\partial \mathbf{g}}{\partial \mathbf{z}}$ will be attended with deterioration in condition of matrices in (10). The alternative is the usage for calculations of original linearized descriptor model (7), (8). 


\section{Invariant zeros definition}

Let us introduce the following notation of block vector and matrix:

$$
\begin{gathered}
x=\left(\begin{array}{c}
x^{\prime} \\
\hdashline x^{\prime \prime}
\end{array}\right) \in \mathbf{R}^{n}, \boldsymbol{E}=\left(\begin{array}{c:c}
\boldsymbol{E}_{n_{x}} & 0 \\
\hdashline 0 & 0
\end{array}\right) \in \mathbf{R}^{n \times n}, \boldsymbol{A}=\left(\begin{array}{c:c}
\frac{\partial \mathbf{f}}{\partial \mathbf{x}} & \frac{\partial \mathbf{f}}{\partial \mathbf{z}} \\
\frac{\partial \mathbf{g}}{\partial \mathbf{x}} & \frac{\partial \mathbf{g}}{\partial \mathbf{z}}
\end{array}\right) \in \mathbf{R}^{n \times n}, \\
\boldsymbol{B}=\left(\begin{array}{c}
\frac{\partial \mathbf{f}}{\partial \mathbf{u}} \\
\frac{\partial \mathbf{g}}{\partial \mathbf{u}}
\end{array}\right) \in \mathbf{R}^{n \times r}, \boldsymbol{C}=\frac{\partial \mathbf{h}}{\partial \mathbf{x}}: \frac{\partial \mathbf{h}}{\partial \mathbf{z}} \in \mathbf{R}^{m \times n},
\end{gathered}
$$

where $\boldsymbol{E}_{n_{x}}-$ identity matrix of order $n_{x} \times n_{x}$; $n=n_{x}+n_{z}$. Then descriptor system (7)-(8) can be written over in more standard form

$$
\boldsymbol{E} \frac{d x}{d t}(t)=\boldsymbol{A} x(t)+\boldsymbol{B} u(t), y(t)=\boldsymbol{C} x(t),
$$

where $x(t) \in \mathbf{R}^{n}$ - state vector, $u(t) \in \mathbf{R}^{r}$ - input vector, $y(t) \in \mathbf{R}^{m}$ - output vector.

Define:

Definition [1]. Complex frequency $\lambda=\alpha^{*}$, where column rank of matrix

$$
\left(\begin{array}{c:c}
\boldsymbol{A}-\alpha^{*} \boldsymbol{E} & \boldsymbol{B} \\
\hdashline \boldsymbol{C} & 0
\end{array}\right)
$$

Is decreasing, is called invariant zero.

In (13) $\lambda \in \mathbf{C}$ - complex variable; $\mathbf{0}$ - zero matrix of order $m \times r ; \mathbf{C}-$ complex plane.

Invariant zeros are defined from the condition [10]

$$
\left(\begin{array}{c:c}
\boldsymbol{A}-\alpha^{*} \boldsymbol{E} & \boldsymbol{B} \\
\hdashline \boldsymbol{C} & 0
\end{array}\right)\left(\begin{array}{l}
x_{0} \\
\hdashline u_{0}
\end{array}\right)=0
$$

of full «blocking» of signal transmission both eigen component and forced component of dynamic motion, i.e. for $\lambda=\alpha^{*}$ there are such vectors $x_{0}$ и $u_{0}$, that system output reaction equal to zero. Thus, the problem of determining of invariant zeros of the system (12) resolves oneself into eigenvalue generalized problem in the general case of rectangular pencil matrix

$$
\left(\begin{array}{c:c}
\boldsymbol{A}-\lambda \boldsymbol{E} & \boldsymbol{B} \\
\hdashline \boldsymbol{C} & 0
\end{array}\right) .
$$

The search of Invariant zeros for large dynamic systems $(n] 1)$ comes across number of significant calculation difficulties. Calculation errors and calculation problems of zeros of generalized pencil matrices [11] play a crucial role in here. Therefore new approaches to development of computing algorithms are needed.

\section{Randomized squaring}

Rule of thumb calculation of invariant zeros of descriptor system (12) connected with so called dynamic system squaring up.

Suppose $m>r$ (number of outputs of a system is more that number of inputs). Let us introduce into consideration the descriptor system

$$
\boldsymbol{E} \frac{d x}{d t}(t)=\boldsymbol{A} x(t)+\boldsymbol{B} u(t), y^{*}(t)=\boldsymbol{T}_{C} \boldsymbol{C} x(t),
$$

where $\boldsymbol{T}_{C} \in \mathbf{R}^{r \times m}$ - full row rank matrix.

The assertion, driven by verbal proof, is valid similar to [9].

Theorem 1. Set of invariant zeros of descriptor system (12) is always a subset of a set of invariant zeros of squared descriptor system (16), but not vice versa.

Suppose hereafter $r>m$ (number of inputs of a system is more that number of outputs). Consider the descriptor system

$$
\boldsymbol{E} \frac{d x}{d t}(t)=\boldsymbol{A} x(t)+\boldsymbol{B} \boldsymbol{T}_{B} u^{*}(t), y(t)=\boldsymbol{C} x(t),
$$

where $\boldsymbol{T}_{B} \in \mathbf{R}^{m \times r}$ - full column rank matrix.

The assertion dual to theorem 1 is valid.

Theorem 2. Set of invariant zeros of descriptor system (12) is always a subset of a set of invariant zeros of squared descriptor system (17), but not vice versa.

Lemmas 1, 2 Simply, that squaring operation generally puts into the system new zeros. This must be considered for choice of $\boldsymbol{T}_{C}$ and $\boldsymbol{T}_{B}$ matrices. The randomisation procedure, proposed by authors, is one of methods of solution for a choice of $\boldsymbol{T}_{C}$ and $\boldsymbol{T}_{B}$ matrices.

Let us introduce into consideration randomization operator Rand ${ }_{r \times m}$, which generates full rank matrices of order $r \times m$ with normal law distributed elements. Also suppose Orth $_{r \times m}-$ orthogonalization operator for full rank matrix of order $r \times m$.Then

$$
\text { Orth }_{r \times m} \text { Rand }_{r \times m}
$$

- operators composition, which action result is a randomized orthogonalization full rank matrix.

Let us also specify $\Omega$ - set of invariant zeros of the system (12), $\Omega^{*}$ - set of invariant zeros of squared descriptor system. To be specific hereafter it's as if $m>r$ and и squared descriptor system is of the form of (16).

Suppose $\Omega_{i}^{*}, i=1,2, \ldots-$ sets of invariant zeros of squared descriptor systems, where

$$
\boldsymbol{T}_{C}^{(i)}=\text { Orth }_{r \times m} \text { Rand }_{r \times m} .
$$

On the analogy of [12] it may be shown, that intersection of all sets of invariant zeros of squared systems $\Omega_{i}^{*}, i=1,2, \ldots$, coincides with the set of invariant zeros of the given system $\Omega$.

\section{Evaluation of invariant zeros of large electrical power system}

Let us find the solution of evaluation problem of invariant zeros of large electrical power system, composed of 25 electrical power plants. Mathematical model of electrical power system in phase coordinates «phase angle - generator slip» in form (12) has matrices of following orders:

$$
A \in \mathbf{R}^{50 \times 50}, B \in \mathbf{R}^{50 \times 25}, C \in \mathbf{R}^{20 \times 50}, E \in \mathbf{R}^{50 \times 50} .
$$


Portrait of Jacobi matrix $\boldsymbol{A}$ is shown on fig. 1. To put it differently, there is control system on each of 25 power stations. Measurement of phase coordinates is performed on-line only on 20 power plants with synchronized vector meters included in mode parameters monitoring system.

Thus, pencil matrix (15) is rectangular and has order of $70 \times 75$ in this case. By using orthogonalization and randomization operators let us generate a set

$$
\boldsymbol{T}_{i},=1, \ldots, k=\text { Orth }_{25 \times 20} \operatorname{Rand}_{25 \times 20},
$$

and then construct square pencil matrices

$$
\left(\begin{array}{c:c}
\boldsymbol{A}-\lambda \boldsymbol{E}_{50} & \boldsymbol{B \boldsymbol { T } _ { i }} \\
\hdashline \boldsymbol{C} & \mathbf{0}_{20 \times 20}
\end{array}\right), i=1, \ldots, k . .
$$

Matrix eigenvalues computational results (22) for $k=500$ (i.e. 15000 eigenvalues) in form of their generalized portrait are shown on fig.1.

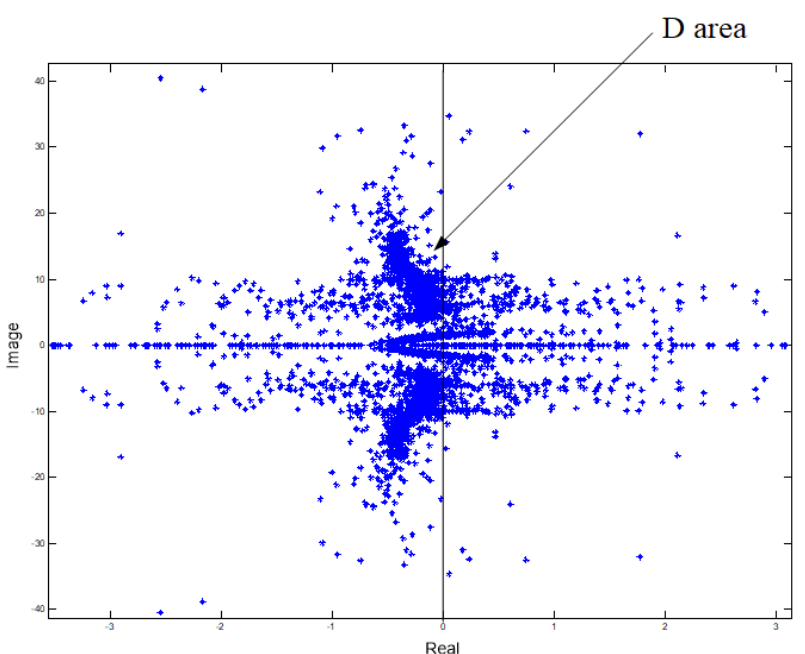

Fig. 1 - Eigenvalues of matrices (22)

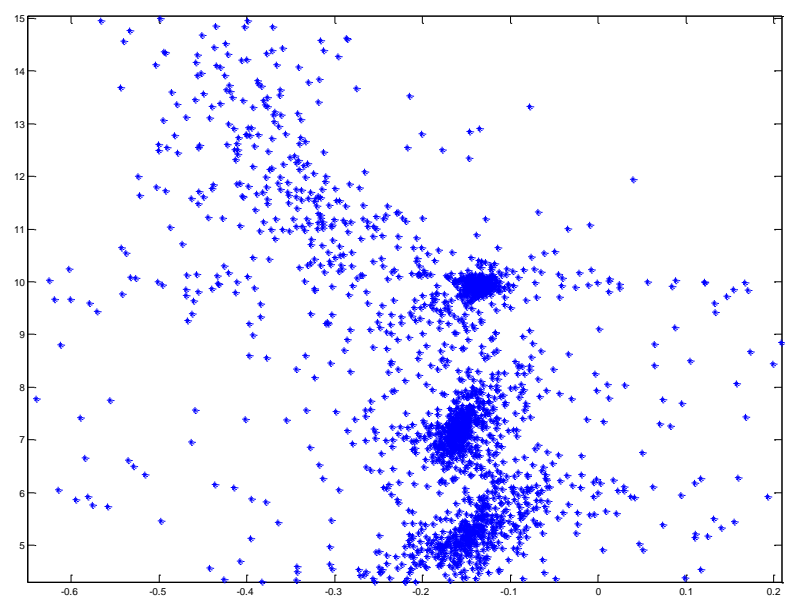

Fig. 2 - Eigenvalues of matrices (22) in D area

Research of generalized portrait shows that there are three groups of tight accumulations in D area, which could be characterized as invariant zeros candidates. Accumulation centres match up complex frequencies, on which the matrix (15) of considered electrical power system model has close to zero minimal singular values, i.e. the matrix is close to loss of its rank. This is the practical invariant zero «indicator» $\alpha^{*}$.Thus, considered complex frequencies can be undertaken as electrical power system invariant zeros (20).

From physical standpoint estimated invariant zeros match up modal forms (mode) of oscillations, on which «blocking» of analyzed electrical energy system is happens. This is reflected in the fact that, firstly, it is impossible to reestablish conditions of other 5 power plants, by the information from phasor measurement units (PMU), installed as was shown before on 20 power plants. Secondly, the sudden loss of efficiency of stabilizing PI and PID controls, which use the given signals of mode parameters monitoring system.

\section{Conclusion}

Invariant zeros directly connects with controllability and observability of dynamic system, including principle possibility of PI, PD and PID control law synthesis and realisation and servocontrol as well. Analysis of Invariant zeros of large dynamic systems, to which modern electrical power systems belong, comes across number of significant calculation difficulties, where calculation errors and calculation problems of zeros of generalized pencil matrices play a crucial role. Thus, new approaches to development of computing algorithms are needed.

The randomized squaring procedure is proposed in the report, allows calculating efficiently invariant zeros of dynamic system, given in descriptor form.

Invariant zeros can be matched up with modes of oscillations, on which «blocking» of analyzed electrical energy system is happens, that is reflected in the fact that, it is impossible to reestablish conditions by the information from synchronized phasor measurement units of electrical power system elements (loss of full observability) and due to the sudden loss of efficiency of stabilizing PI and PID controls, which use given signals for electrical power system stabilization (loss of full controllability).

\section{References}

1. Brockett R.W., IEEE Trans. Automat. Control,1965, 10. pp. $129-135$.

2. Rosenbrock H.H. State space and multivariable theory. (Nelson-Wiley, 1970).

3. Zhou K. Essentials of Robust Control. Prentice Hall, 1998.

4. Zubov N.E., Mikrin E.A., Ryabchenko V.N. Matrix approach in theory and practice of automatic flight-control system (Bauman Moscow State Technical University Publ., Moscow 2016).

5. Zubov N.E., Mikrin E.A., Misrikhanov M, Sh, Ryabchenko V.N., Journal of Computer and Systems Sciences International. 2018, 9, pp. $141-151$.

6. Misrikhanov M, Sh, Ryabchenko V.N., Avtomatika i Telemkhanika [Automatics and teleautomatic]. 2008, 10, pp. 31-47. (in Russian)

7. Smagina E,M. Calculation and specification of zeros of a linear multidimensional system. Avtomatika i 
Telemkhanika [Automatics and teleautomatic], 1987, 12. pp. 165 - 173. (in Russian)

8. Zubov N.E., Mikrin E.A., Misrikhanov M, Sh, Ryabchenko V.N., Journal of Computer and Systems Sciences International, 2018. 1, pp. 20 - 26.

9. Macfarlane A.G.J., Kareanias N. Pole and zeros of linear multivariable systems: a survey of the algebraic, geometric and complex variable theory. Int. J. Contr. 1976. Vol. 24, No 1. pp. 33-74.

10. Smagina E.M. Analysis matters of linear multidimensional objects using the concept of zero of a system. Tomsk, Tomsk Univeersity Publ., 1990.

11. Misrikhanov M, Sh, Ryabchenko V.N., Avtomatika i Telemkhanika [Automatics and teleautomatic]. 2006, 5. 24 - 47. (in Russian)

12. Granichin O.N., Polyak B.T. Randomized algorithms for estimation and optimization under almost arbitrary disturbances. Moscow, Nauka Publ., 2003. 